\title{
TAMAÑO ÓPTIMO DE MUESTRA PARA EVALUAR EL PATRÓN DE CRECIMIENTO DE FRUTOS DE NARANJO 'VALENCIA LATE'
}

\author{
MARÍA MERCEDES AVANZA², SERGIO JORGE BRAMARDI ${ }^{3}$, SILVIA MATILDE MAZZA ${ }^{4}$
}

RESUMEN - Los modelos de crecimiento de frutos describen la evolución de su tamaño a lo largo del -período de desarrollo. Con fines de pronóstico, estos modelos permiten estimar en forma anticipada el tamaño que alcanzarán los frutos al momento de la cosecha. Para lograr estimaciones insesgadas del tamaño de frutos a cosecha es necesario un diseño adecuado de muestreo en la etapa de recolección de datos. El objetivo del presente trabajo fue determinar el tamaño óptimo de muestra, compuesta por árboles $(n)$ y frutos $(m)$, para establecer modelos de crecimiento de frutos de naranjo 'Valencia late', que permitan estimar la distribución de tamaño a la cosecha. Se trabajó con el diámetro ecuatorial de frutos previo a la cosecha, proveniente de dos huertos comerciales ubicados en la provincia de Corrientes, Argentina, durante tres temporadas. Mediante modelos mixtos se estimaron las componentes de varianzas entre árboles y frutos, y posteriormente a partir de dos tipos de metodologías se determinó el tamaño de muestra óptimo. La variabilidad entre frutos fue superior a la variabilidad entre árboles. Para la determinación del patrón de crecimiento de frutos de naranjo 'Valencia late' mediante un muestreo bietápico, se sugiere seleccionar 7 árboles y 30 frutos de cada árbol, para lograr estimaciones del diámetro ecuatorial de frutos con una precisión entre el 2 y $3 \%$.

Términos para indexación: biometría, curvas de crecimiento, frutos cítricos, muestreo de frutos.

\section{OPTIMAL SAMPLE SIZE FOR EVALUATE THE GROWTH PATTERN OF 'VALENCIA LATE' ORANGE FRUIT}

\begin{abstract}
Fruit growth models are used, among other applications, to describe the evolution of fruit size throughout its development period. For forecasting, these models allow estimate in advance the size that will achieve the fruits at harvest. To have growth curves that provide unbiased estimate of the fruit size at harvest is necessary to design an appropriate sampling at the data collection stage for its construction. The objective of the present work was to determine the optimal sample size, consisting of trees $(n)$ and fruits $(m)$, to establish the growth patterns of orange fruits 'Valencia late' to estimate the size distribution at harvest. It was used the equatorial diameter of fruits before harvest, from two orchards located in the province of Corrientes, Argentina, during three seasons. Through mixed models were estimated components of variances between trees and fruits, and then from two types of methodologies was determined optimal sample size. The variability among fruits was higher than the variability between trees. To determine the growth pattern of orange fruit 'Valencia late' by sampling in two-stages, it is suggested to select 7 trees and 30 fruit per tree, for the estimative to reach equatorial diameter of fruit with an accuracy between 2 and $3 \%$.
\end{abstract}

Index terms: biometric, citrus fruit, fruit sampling, growth curves.

\footnotetext{
${ }^{1}$ (Trabalho 226-09). Recebido em: 30-09-2009. Aceito para publicação em: 22-04-2010.

${ }^{2}$ Ing. Agr. Facultad de Ciencias Agrarias (FCA). Universidad Nacional del Nordeste (UNNE). Sargento Cabral 2131. (3400) Corrientes. Argentina.mavanza@agr.unne.edu.ar

${ }^{3}$ Ing. Agr. Dr. Facultad de Ciencias Agrarias y Forestales. Universidad Nacional de La Plata. Calle 60 y 119. (1900) La Plata. Buenos Aires. Argentina. bramardi@arnet.com.ar

${ }^{4}$ Ing. Agr. Dra. FCA - UNNE. Sargento Cabral 2131. (3400) Corrientes. Argentina. smmazza@unne.edu.ar
} 


\section{INTRODUCCIÓN}

En modelos de predicción de producción de frutos, varios parámetros relativos a características que los mismos presentarán a la fecha de ser recolectados deben ser estimados anticipadamente. Uno de ellos, de fundamental importancia por su incidencia en la precisión de los volúmenes predichos, es el tamaño que los frutos tendrán al final del período de fructificación. La elaboración de curvas de crecimiento permite conocer el modo en que crece el fruto con respecto al tiempo y los modelos estadísticos ajustados a dichas curvas se utilizan, entre otras aplicaciones, para estimar en forma anticipada el tamaño que se espera alcancen los frutos al momento de la cosecha y de esta manera realizar pronósticos de producción (BRAMARDI, 1995; ÁLVAREZ et al., 1999; CUEVAS et al., 2003; AVANZA et al., 2008).

En todo experimento o investigación agronómica se alcanza un punto en el cual se debe tomar una decisión acerca del tamaño de muestra. Una muestra demasiado grande implica un desperdicio de recursos y una muestra demasiado pequeña disminuye la utilidad de los resultados de las estimaciones y pruebas de hipótesis. El problema principal al momento de determinar el tamaño de una muestra es obtener alta precisión y bajo costo para la estimación de un determinado parámetro. La precisión puede establecerse por el recíproco de la estimación de varianza, mientras que los costos se basan principalmente en el tiempo empleado en la toma de mediciones. Dicha precisión puede ser prefijada por el investigador, mientras que el costo en tiempo puede disminuirse tomando aquel tamaño de muestra mínimo que proporcione la precisión deseada (COCHRAN, 1982; GOMEZ; GOMEZ, 1984; SCHEAFFER et al., 2007).

Diversos autores proponen diferentes criterios para la determinación de tamaño de muestra aplicados a estudios agronómicos, entre los cuales se puede mencionar a: Demey et al. (2004), los cuales utilizaron gráficos de funciones entre errores estándar y los diferentes tamaños de muestras en experimentos biotecnológicos de caña de azúcar, y consideraron el tamaño óptimo de muestra por medio del mínimo relativo de la función. Rozane et al. (2007), con el fin de determinar la cantidad de hojas de mango necesarias para el análisis de macro y micronutrientes, proponen el cálculo del porcentaje de error en relación a la media para cada tamaño de muestra, con un error admisible entre 5 y $10 \%$. Para determinar el número de plantas adecuado para estimar poblaciones de ácaro de la lepra en plantaciones de naranja
Valencia, Vilela Lapes et al. (2007) calcularon el error estándar de la media para diferentes tamaños de muestras de una proporción, y concluyeron que es necesario muestrear un mayor número de plantas a la cantidad utilizada comúnmente para obtener un error de estimación medio aceptable del $25 \%$.

Todos los trabajos citados anteriormente se basan en calcular tamaños de muestra para un diseño de muestreo aleatorio simple y utilizan como criterio de selección del tamaño óptimo, un error máximo admitido, expresado en relación al promedio o determinado a partir del error estándar u otra medida de variabilidad.

Una de las dificultades en estudios del crecimiento u otros atributos de frutos, es la selección de una estrategia de muestreo apropiada para estimar el tamaño de frutos teniendo en cuenta la variación dentro de cada árbol (MILES et al. 1996; DE SILVA; BALL, 1997; DE SILVA et al., 2000; OPARA, 2000).

La variabilidad del carácter bajo estudio puede ser calculada a través de diferentes métodos de estimación. Los modelos de componentes de varianza son modelos lineales que incorporan términos aleatorios, lo cual genera una matriz de varianzas y covarianzas con estructura conocida pero con valores paramétricos desconocidos que deben ser estimados (MONTGOMERY, 1981; CADENAS-MENESES; CASTILLO MORALES, 2000).

En este aspecto, Miles et al. (1996), De Silva e Ball (1997) y De Silva et al. (2000), han estudiado la variabilidad de distintas características de frutos de kiwi y manzana, entre diferentes huertos y sectores dentro de los mismos; y basándose en este conocimiento, han diseñado estrategias de muestreo y comparación de la exactitud y eficiencia en la estimación de la media. De Silva e Ball (1997), De Silva et al. (2000), utilizaron modelos mixtos para describir el patrón de variabilidad y dependencia espacial del peso de frutos de kiwi y manzana. La fuente predominante de variación del peso fue dada entre frutos. A partir de dichos resultados proponen planes de muestreo sistemático los cuáles proporcionarán un estimador más eficiente e insesgado.

$\mathrm{Al}$ momento de muestrear frutos en huertos comerciales para el estudio de curvas de crecimiento, se plantea la necesidad de diseñar un muestreo que consta de dos etapas (muestreo bietápico), donde es necesario realizar una primera selección de árboles y luego una segunda etapa de muestreo de frutos dentro de cada árbol. En este sentido, Bramardi (1995), para estimar el tamaño final de frutos de peras Packham `s Triumph y William 's en la región del Alto Valle de Río Negro y Neuquén, Argentina, determinó el tamaño óptimo de unidades primarias (árboles) y unidades 
secundarias (frutos) mediante las expresiones dadas por Cochran (1982).

El objetivo del presente trabajo fue determinar el tamaño óptimo de muestra, compuesta por árboles $\mathrm{y}$ frutos, para establecer modelos de crecimiento de frutos de naranjo 'Valencia late', que permitan estimar la distribución de tamaño a la cosecha.

\section{MATERIALES Y MÉTODOS}

\section{Datos experimentales}

La toma de datos se realizó en dos huertos comerciales de naranjo dulce [Citrus sinensis (L.) Osbeck] 'Valencia late' injertados sobre lima de Rangpur (Citrus limonia, Osbeck), ubicados en el departamento de Concepción ( $\left.28^{\circ} 16^{`} \mathrm{~S}, 58^{\circ} 05^{`} \mathrm{O}\right)$ (huerto 1) y en el departamento de General Paz (27 $45^{`} \mathrm{~S}, 57^{\circ} 37^{`} \mathrm{O}$ ) (huerto 2), de la provincia de Corrientes, Argentina, durante las temporadas 2003-04 (1), 2004-05 (2) y 2005-06 (3).

El lote seleccionado en el huerto 1 está constituido por árboles de 12 años de edad, una densidad de 357 árboles $\mathrm{ha}^{-1}$, y un marco de plantación de $4 \times 7 \mathrm{~m}$; y el lote del huerto 2 por árboles de 27 años, densidad de 312 árboles ha ${ }^{-1}$, marco de plantación de $4 \times 8 \mathrm{~m}$. Ambos huertos presentan una condición productiva media y buen estado sanitario, manejados con prácticas culturales habituales de la región.

$\mathrm{Al}$ inicio de cada temporada, en cada huerto se seleccionaron al azar árboles y dentro de cada árbol frutos distribuidos en toda la copa, en los cuáles de determinó el diámetro ecuatorial ( $\mathrm{mm}$ ) semanalmente con calibre digital. En la Tabla 1, se muestra el número de árboles y frutos de la última fecha de medición cercana a la cosecha, los cuáles fueron utilizados en el presente trabajo.

Se decidió tomar la información de la última fecha de medición de cada huerto y temporada siguiendo el trabajo de Bramardi (1995), el cuál comprobó que la máxima variabilidad del diámetro ecuatorial de frutos de pera se presenta al final del período de crecimiento de los frutos, en el período próximo a la cosecha y que las variabilidades absolutas serán mayores cuanto más grandes sean los frutos.

\section{Estimación de variabilidad}

Como paso previo a la determinación del tamaño óptimo de muestra se requiere conocer la variabilidad del diámetro ecuatorial de fruto al momento de la cosecha tanto entre árboles como entre frutos.

Una alternativa es plantear el modelo de análisis correspondiente al muestreo y luego en función de las esperanzas de los distintos cuadrados medios involucrados, estimar la variancia del diámetro medio de fruto entre frutos como entre árboles (BRAMARDI, 1995).

El modelo lineal asociado fue el siguiente:

$$
y_{l(i j k)}=\mu+t_{i}+h_{j}+t * h_{i j}+a_{k(i j)}+\varepsilon_{l(i j k)}
$$

$y_{l(i j k)}=$ diámetro ecuatorial $(\mathrm{mm})$ del fruto 1 , correspondiente al árbol $\mathrm{k}$, huerto $\mathrm{j}$ temporada $\mathrm{i}$.

$\mu=$ media general

$t_{i}=$ efecto correspondiente a la i-ésima temporada.

$h_{j}=$ efecto correspondiente al j-ésimo huerto.

$t^{*} h_{j i}=$ efecto correspondiente a la interacción entre la i-ésima temporada y el j-ésimo huerto.

$a_{k(i j)}=$ efecto del k-ésimo árbol de la temporada i y huerto $\mathrm{j}$.

$\varepsilon_{l(i j k)}$ efecto correspondiente al fruto 1 , del árbol i, huerto $\mathrm{j}$ y temporada $\mathrm{i}$.

Los efectos correspondientes a árbol y fruto se consideraron aleatorios debido al proceso de selección de los mismos, y los efectos de huerto y temporada fijos. En cada combinación huerto-temporada, los árboles seleccionados fueron diferentes, por lo que el factor árbol se anida dentro de la interacción en el modelo.

Para la estimación de las componentes de varianzas del modelo propuesto en Eq. (1), se utilizó el procedimiento MIXED del software estadístico SAS (SAS INSTITUTE, 2000). El procedimiento MIXED ajusta modelos mixtos, lo cuales son una extensión del modelo lineal general, y pueden proporcionar estimaciones y hacer inferencias tanto de efectos fijos como aleatorios. Se utilizó el método de máxima verosimilitud restringida (REML), para la estimación de parámetros desconocidos del modelo (SAS INSTITUTE, 2004).

Determinación del tamaño óptimo de muestra

1. Mediante la metodología para muestreo en dos etapas, propuesta por Cochran (1982), se calculó el tamaño óptimo de muestra de árboles $(n)$ y frutos $(m)$ para el diámetro ecuatorial. La forma habitual de determinar el tamaño muestral óptimo consiste, ya sea en fijar un nivel de precisión a conseguir en las inferencias, o bien en maximizar o minimizar alguna función objetivo que refleja la utilidad de resolver satisfactoriamente el análisis. Esta función objetivo generalmente viene asociada a los costos o trabajo involucrado para relevar unidades primarias $\left(c_{n}\right)$ frente a unidades secundarias $\left(c_{m}\right)$. El $m$ y $n$ óptimos se calculan minimizando los costos para una varianza fija con las siguientes expresiones: 


$$
m=\sqrt{\frac{S_{m}^{2} \cdot c_{n}}{S_{n}^{2} \cdot c_{m}}} \quad(2) \quad n=\frac{m \cdot s_{n}^{2}+s_{m}^{2}}{m V \cdot(\bar{y})}
$$

Donde:

$S_{n}^{2}=$ estimación de varianza entre unidades primarias (árboles);

$S_{m}{ }^{2}=$ estimación de varianza entre unidades secundarias (frutos);

$c_{n}=$ costo de muestrear unidades primarias (horas hombre, expresado en minutos);

$c_{m}=$ costo de muestrear unidades secundarias (horas hombre, expresado en minutos);

$\mathrm{Cn} / \mathrm{Cm}=$ relación entre el costo de muestrear unidades primarias y el costo de muestrear unidades secundarias (horas hombre que implica identificar una unidad primaria respecto a una unidad secundaria); $V(\bar{y})=$ es el error admitido de la media del diámetro ecuatorial. Se utilizó $1 \mathrm{~mm}$ que es la diferencia del diámetro medio entre dos tamaños comerciales sucesivos más cercanos (calibres comerciales 125 y 130, que expresan el número de frutos por caja telescópica de $15 \mathrm{~kg}$ ).

Para obtener una estimación de la relación entre costos de muestrear unidades primarias y unidades secundarias, se midieron las horas-hombre que implicaba muestrear y marcar un árbol y horashombre insumidas en la selección y marcado de un fruto, resultando 3,95 minutos para árbol $\left(c_{n}\right)$ y 0,86 minutos para fruto $\left(c_{m}\right)$, en consecuencia la relación entre los costos $\left(c_{n} / c_{m}\right)$ fue 4,59.

2. Por otro lado, se propone una metodología diferente, basada en la variación de los porcentajes de error en relación a la media $\left(\hat{\delta}_{i}\right)$, al incrementarse el tamaño de muestra.

Se calcularon y graficaron dichos errores para las diferentes combinaciones de $m$ y $n$, a partir de las siguientes expresiones:

$$
\begin{gathered}
\hat{\delta}_{i}=Z_{5 \%}\left[\frac{1}{n} \cdot S_{n}^{2}+\frac{1}{m} \cdot S_{m(n)}^{2}\right]^{1 / 2} \\
\hat{\delta}_{i}^{\prime}(\%)=100 \cdot \frac{\hat{\delta_{i}}}{y_{i}}
\end{gathered}
$$

$\hat{\delta}_{i}=$ precisión absoluta o semiamplitud del intervalo de confianza (margen de error permitido en la estimación de la media del diámetro de frutos).

$\mathrm{Z}_{5 \%}=$ valor de la distribución normal estándar $(\alpha=0,05)$.

$\mathrm{S}^{2}=$ estimación de varianza entre árboles $(n)$.

$\mathrm{S}^{2}{ }_{m(n)}^{n}=$ estimación de varianza entre frutos del árbol $n$.

$\hat{\delta}_{i}(\%)=$ precisión relativa expresada en porcentaje de la media del diámetro de frutos.

Para seleccionar el tamaño de muestra óptimo de árboles y frutos, en el gráfico resultante, se buscó el punto en el cuál se logra un error de estimación inferior al $3 \%$, que corresponde a $2 \mathrm{~mm}$ del diámetro ecuatorial de frutos.

\section{RESULTADOS Y DISCUSIÓN}

En la Tabla 2, se observan las estimaciones de media, error estándar (EE), e intervalos de confianza (LI y LS 95\%) para la muestra original del diámetro ecuatorial de frutos de cada huerto y temporada. Se observa que los EE son similares en ambos huertos y temporadas, pero las medias difieren en distintos grados de significancia entre huertos y entre temporadas.

En la Figura 1 se muestra la dispersión del diámetro ecuatorial de frutos por huerto, para las tres temporadas. Se puede observar que el patrón de dispersión es similar entre huertos, pero dentro de huertos se observa la variación respecto a las temporadas, la cuál está dada por las diferentes condiciones ambientales y de manejo presentadas en cada año.

\section{Estimación de variabilidad}

De acuerdo al modelo lineal mixto propuesto en la Eq. (1), la interacción huerto x temporada $\left(t * h_{i j}\right)$, resultó significativa $(\mathrm{p}<0,0001)$. La Figura 2 representa el diámetro promedio de los frutos por temporada para cada huerto y permite identificar el origen de la interacción huerto x temporada, la que está dada principalmente porque en el huerto 1, hay un comportamiento dispar en el tamaño de los frutos a lo largo de las temporadas, mientras que en el huerto 2 esta es más uniforme.

Las estimaciones de varianzas del modelo de la Eq. (1), arrojaron una varianza entre árboles $\left(S_{n}^{2}\right)$ de 3,3776 y entre frutos $\left(S_{m}{ }^{2}\right) 15,6000$. Dichos resultados muestran claramente la predominancia de la componente de variación del diámetro entre frutos sobre la de árboles, alrededor del $82 \%$ de la variación total corresponde a la variabilidad entre frutos. Estos resultados concuerdan con los reportados por Bramardi (1995), el cuál encuentra la mayor variabilidad del diámetro entre frutos y menor variabilidad entre árboles de pera; y De Silva \& Ball (1997) y De Silva et al. (2000), los cuales encontraron predominancia de variabilidad del peso de frutos de kiwi y manzana entre los mismos. Por otro lado, Miles et al. (1996), hallaron que la mayor variabilidad del peso de frutos de kiwi dentro de zonas de una planta, seguido 
por la variabilidad entre plantas, y generalmente, la variación entre las partes de las plantas fue un $2 \%$ inferior que la variación entre plantas.

Las estimaciones de varianza de árboles y frutos por huerto y temporada, no presentan diferencias entre huertos, y si muestran algunas diferencias entre temporadas. Tal como se puede observar en la Figura 1, para el huerto 1 la mayor variabilidad, tanto entre árboles como entre frutos, se presenta en la temporada 1 ; en cambio, para el huerto 2, la mayor variabilidad se observa en la temporada 3 . Se considera que, tanto para árbol como para fruto, las variabilidades del modelo incluyen estas variaciones particulares, y se propone utilizarlas como estimaciones de varianza en la determinación de tamaños de muestra.

Determinación del tamaño óptimo de muestra En la Tabla 3, se presentan los tamaños de muestras $n$ y $m$ obtenidos a partir de las Eq. (2) y (3), en las cuales se utilizaron las estimaciones de varianza de árbol y frutos calculadas previamente. Los resultados indican que se deberían seleccionar 7 árboles $(n)$ con 5 frutos por árbol $(m)$, tomando como error admitido de la media del diámetro ecuatorial de frutos $(V(\bar{y}))$ de $1 \mathrm{~mm}$ y con una relación de costos $\left(c_{n} / c_{m}\right)$ de 4,59.

Por otro lado, en la Figura 3, se aprecia la dispersión del error $\left(\hat{\delta}_{i} \%\right)$ cometido en la estimación de la media del diámetro calculado a partir de Eq. (5), como función del tamaño de muestra compuesto por árboles y frutos. La flecha indica el punto del tamaño de muestra a partir del cual el $\hat{\delta}_{i}^{\prime}(\%)$ se estabiliza. Dicho punto se encuentra entre $2 \%$ y $3 \%$ de error de estimación de la media lo cual equivale a 1 y 2 $\mathrm{mm}$. Dentro de esa franja, se observa que a partir de $n$ variando entre 7 y 8 árboles y $m$ variando entre 30 y 40 frutos por árbol, el $\hat{\delta}_{i}^{\prime}(\%)$ mantiene estable, indicando el punto de equilibrio entre alta precisión de las estimaciones a bajo costo. Esta relación disminuye a medida que aumenta el tamaño de muestra, coincidiendo con los trabajos realizados por Miles et al. (1996), los cuales han diseñado estrategias de muestreo para estimar atributos de calidad de frutos de kiwi al momento de la cosecha, Demey et al. (2004), en la determinación de tamaño de muestra en experimentos biotecnológicos de caña de azúcar, y Rozane et al. (2007), al determinar la cantidad de hojas de mango necesarias para el análisis de macro y micronutrientes.

Con ambas metodologías, la desarrollada por Cochran (1982) (Tabla 3) y la basada en los porcentajes de error en relación a la media $\left(\hat{\delta}_{i}\right)$ (Figura 3 ), se obtienen valores similares para el tamaño de muestra de unidades primarias o árboles $(n=7)$, pero no respecto al tamaño de muestra de unidades secundarias o frutos. De acuerdo a Cochran, se deberían tomar 5 frutos por árbol, el cuál es un valor pequeño comparado con la segunda metodología que arroja valores de $m$ entre 30 y 40 . No obstante se considera más adecuado el número de frutos determinado con la segunda metodología, debido a que el propio Cochran (1982) considera que en la mayoría de las situaciones prácticas el $m$ óptimo resulta relativamente bajo. Por otra parte, la elección de $m$ sugiere las estimaciones $\mathrm{de}_{n} / \mathrm{c}_{m}$ y $\mathrm{S}_{m} / \mathrm{S}$, y resulta difícil obtener estas razones con gran precisión, sobre todo la primera. Si la relación $\mathrm{c}_{n} / \mathrm{c}_{m}$ fuese igual a 20, el tamaño de muestra se modificaría a $n=6$ y $m=10$, lo que duplica el tamaño de muestra de unidades secundarias respecto al valor original manteniendo la misma precisión. Además, si se utiliza la mitad de la diferencia del diámetro entre dos calibres comerciales sucesivos como error admitido $(V(\bar{y})=0,5)$, el número de árboles a seleccionar se duplica, con lo cuál sólo se ganaría una precisión del $0,4 \%$ (Figura 3 ).

Analizando la Figura 3, se puede observar que con valores de $n=7$ y $m=5$ se podría obtener una precisión del $4,40 \%$, el cual no es un mal valor contrastándolo con el \% de precisión sugerida por Miles et al. (1996), en la determinación del tamaño de muestra de frutos de kiwi y por Rozane et al. (2007) en la determinación del tamaño de muestra de hojas de mango; pero se podría disminuir hasta un $2 \%$ seleccionando 30 frutos por árbol $(m=30)$, como lo sugiere la otra propuesta metodológica. En esta última situación, a partir de dicho tamaño de muestra, la precisión se mantiene constante, lo que asegura la selección del tamaño "óptimo" en lo que se podría considerar un punto de equilibrio dado, a partir del cuál el incremento del tamaño de muestra significa mayor esfuerzo y no se corresponde con un incremento significativo en la precisión. 
TABLA 1 - Número de árboles y número promedio de frutos por árbol, por huerto y temporada.

\begin{tabular}{cccc}
\hline Huerto & Temporada & $\mathbf{N}^{\mathbf{0}}$ árboles & $\begin{array}{c}\mathbf{N}^{\mathbf{0}} \text { frutos } \\
\text { promedio . árbol }\end{array}$ \\
\hline \multirow{3}{*}{1} & 1 & 13 & 33 \\
& 2 & 15 & 29 \\
& 3 & 15 & 28 \\
\hline \multirow{2}{*}{2} & 1 & 15 & 18 \\
& 2 & 15 & 23 \\
& 3 & 10 & 31 \\
\hline
\end{tabular}

TABLA 2 - Estimaciones de media, error estándar (EE), e intervalos de confianza de la media (LI y LS) del diámetro ecuatorial $(\mathrm{mm})$ de frutos para cada huerto y temporada.

\begin{tabular}{ccccc}
\hline \multirow{2}{*}{ Huerto } & Estimaciones & \multicolumn{3}{c}{ Temporada } \\
\cline { 3 - 5 } & & $\mathbf{1}$ & $\mathbf{2}$ & $\mathbf{3}$ \\
\hline \multirow{3}{*}{1} & Media & 71,36 & 66,35 & 64,90 \\
& EE & 0,23 & 0,20 & 0,20 \\
& LI - LS (95\%) & $70,91-71,81$ & $65,95-66,76$ & $64,50-65,30$ \\
\hline \multirow{2}{*}{2} & Media & 68,10 & 67,00 & 67.91 \\
& EE & 0,22 & 0,22 & 0,27 \\
& LI - LS (95\%) & $67,67-68,53$ & $66,57-67,43$ & $67,38-68,44$ \\
& & & &
\end{tabular}

TABLA 3 - Determinación del tamaño óptimo de árboles $(n)$ y frutos $(m)$ para el diámetro ecuatorial (mm) por metodología clásica para un muestreo aleatorio bietápico según Cochran (1982).

$$
\begin{aligned}
& \mathrm{S}^{2}=3,3776 \\
& \mathrm{~S}^{2}=15,6000 \\
& c_{n} / c_{m}=4,59 \\
& \boldsymbol{n}=\mathbf{6 , 7 7} \cong \mathbf{7} \\
& \boldsymbol{m}=\mathbf{4 , 6 0} \mathbf{5}
\end{aligned}
$$




\section{Huerto 1}

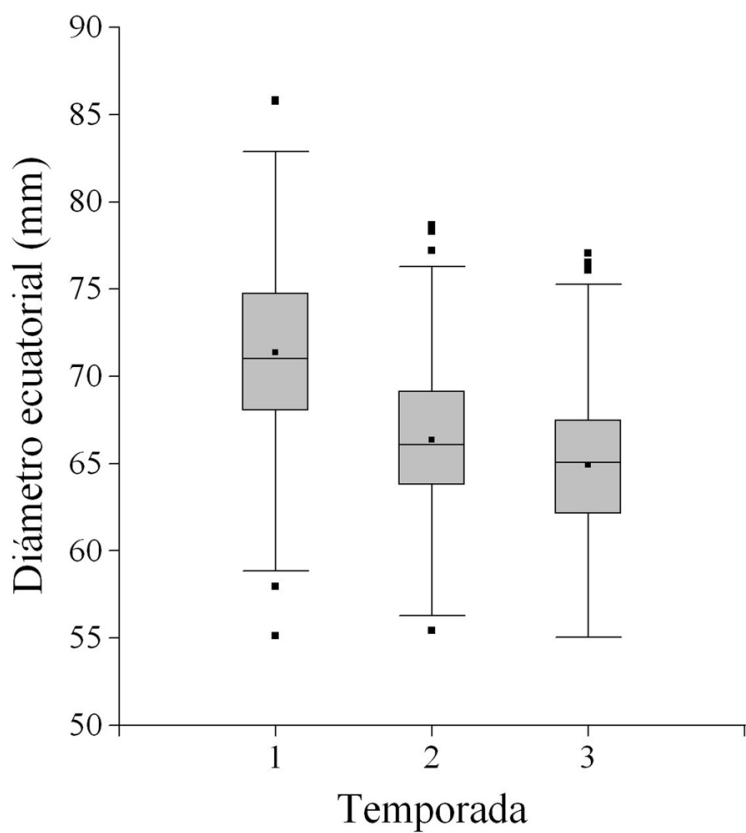

Huerto 2

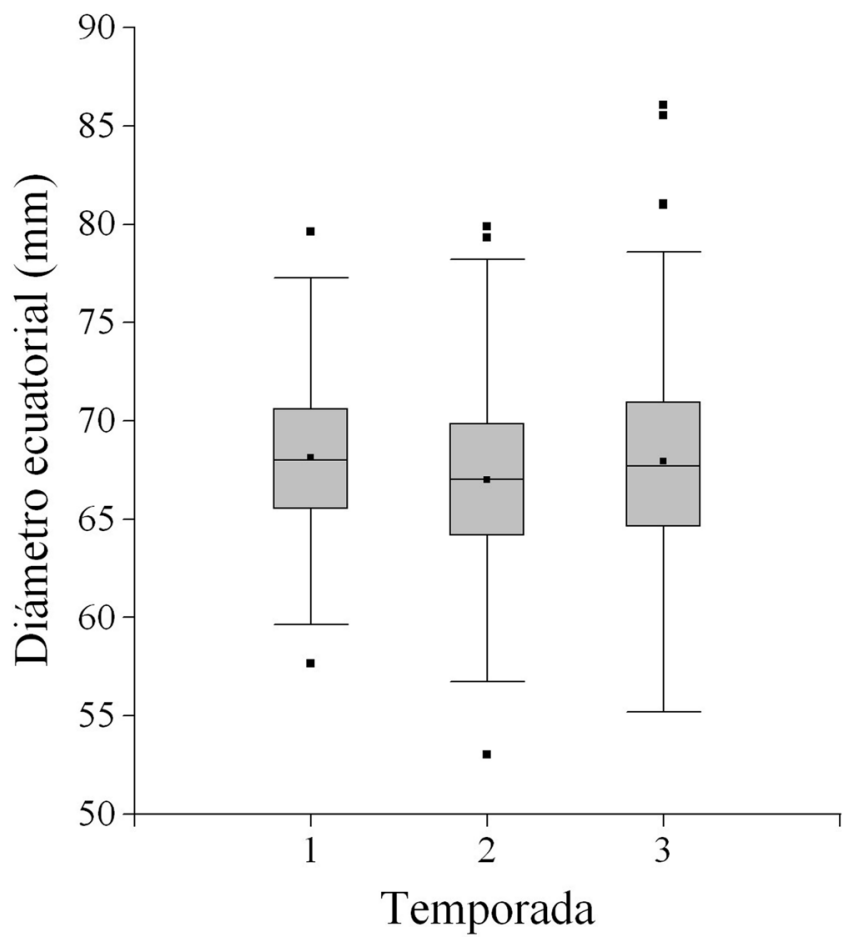

FIGURA 1 - Gráficos de caja del diámetro ecuatorial de frutos por huerto (1-2), para cada temporada. 


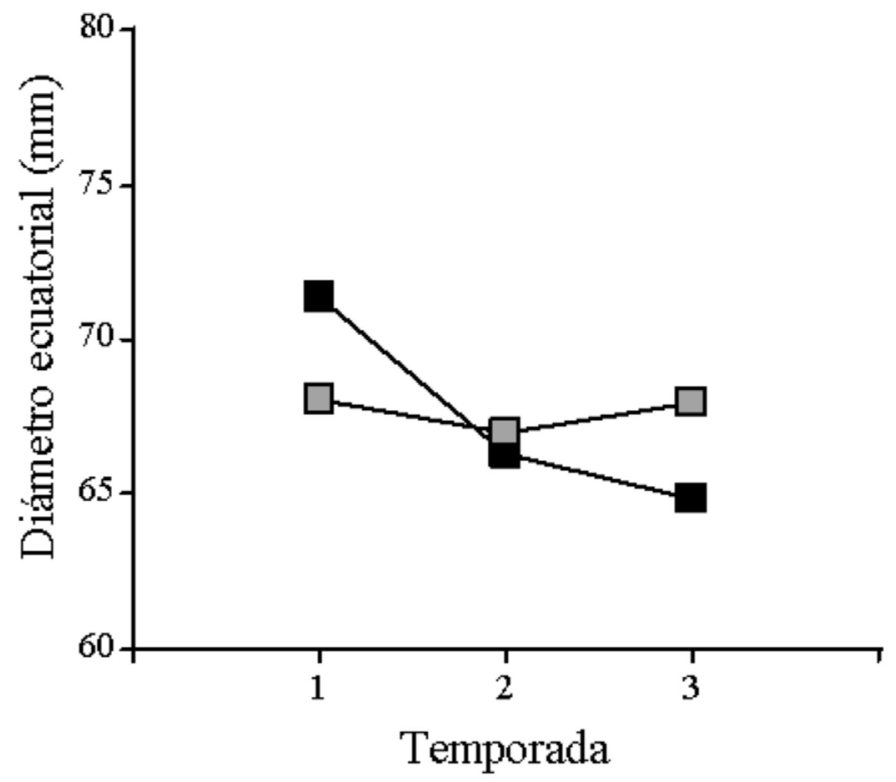

\section{$\longrightarrow$ Huerto $1 \longrightarrow \square-$ Hu erto 2}

FIGURA 2 - Gráfico de puntos indicando el diámetro ecuatorial promedio de frutos por huerto- temporada, y su interacción.

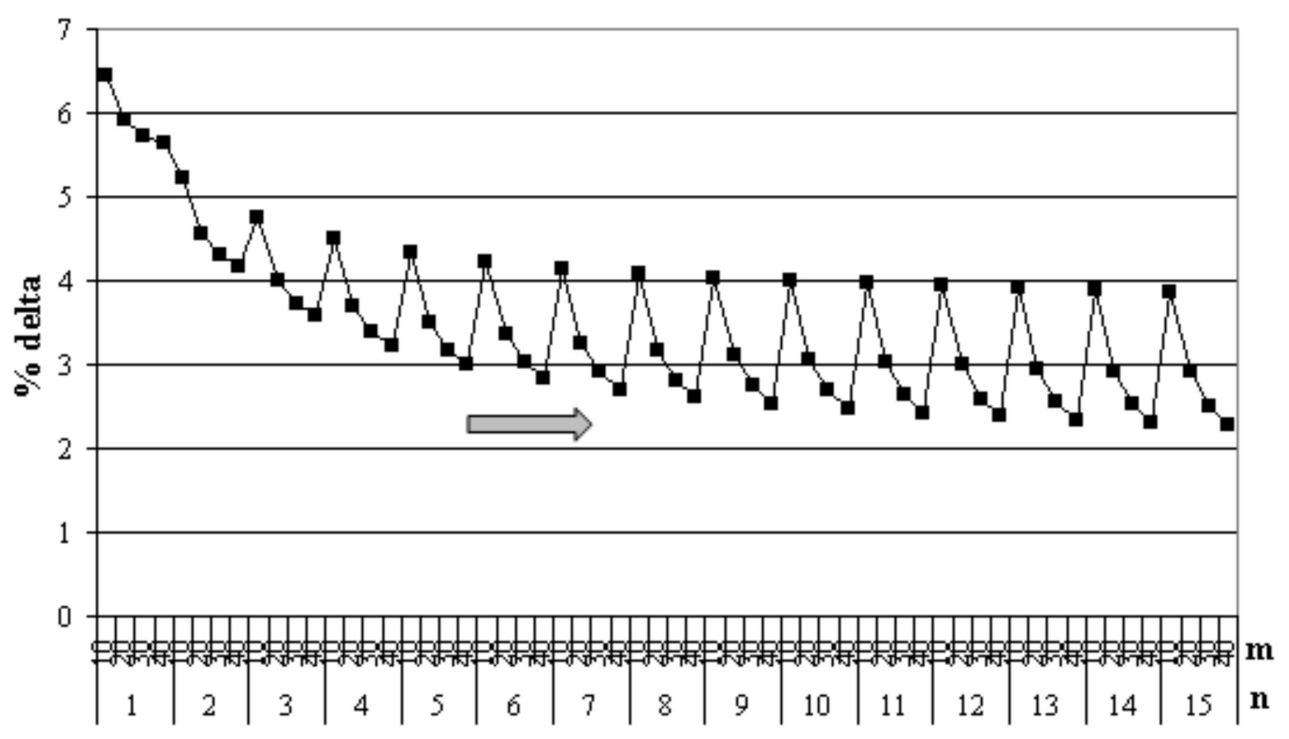

FIGURA 3 - Errores (\% delta) asociados a diferentes tamaños de muestras compuestas por árboles $(n)$ y frutos $(m)$. 


\section{CONCLUSIONES}

1- El $82 \%$ de la variación total del diámetro a la cosecha de frutos de naranjo 'Valencia late' corresponde a la variabilidad entre frutos y el $18 \%$ a la variabilidad entre árboles.

2- Debido a que existe mayor variabilidad del diámetro entre frutos que entre árboles, la relación entre número de frutos a observar por árbol respecto al número de árboles a seleccionar, para un tamaño de muestra total constante, debe ser muy superior a 1 .

3-Para la determinación del patrón de crecimiento de frutos de naranjo 'Valencia late' mediante un muestreo bietápico, se sugiere seleccionar 7 árboles y 30 frutos de cada árbol, para lograr estimaciones del diámetro ecuatorial de frutos con una precisión entre el 2 y $3 \%$.

\section{AGRADECIMIENTOS}

Los autores desean agradecer a los establecimientos Augusto Millán S.A. y Cacique S.A., por ceder gentilmente sus plantaciones citrícolas para la obtención de información para el desarrollo del presente trabajo.

\section{REFERENCIAS}

ÁlVAREZ, A.; BOCHE, S. Modelos matemáticos para describir crecimientos doble sigmoideos en frutos de un nectarin tardío (cv Sun Grand). Agro Sur, Chile, v.27, p. 21-28, 1999.

AVANZA, M.M.; BRAMARDI, S.J.; MAZZA, S.M. Statistical models to describe the fruit growth pattern in sweet orange 'Valencia late'. Spanish Journal of Agricultural Research, Madrid, v.6, n.4, p.577$585,2008$.

BRAMARDI, S.J. Modelos de predicción de tamaño de frutos a la cosecha en base a mediciones sucesivas durante el período de crecimiento. Peras cvs. William`s y Packham`s Triumph en la región del Alto Valle de Río Negro y Neuquén. 1995. Tesis (Magíster Scientiae- Área Biometría) - Facultad de Agronomía, Universidade de Buenos Aires, Buenos Aires, 1995.
CADENA-MENESES, J.A.; CASTILLO-MORALES, A. Una comparación de SAS y Harvey en la estimación de componentes de varianza en modelos mixtos. Agrociencia, México, v. 34, p. 57-68, 2000.

COCHRAN, W.G. Técnicas de muestreo. México: Compañía Editorial Continental, 1982. 513p.

CUEVAS, J.; SALVADOR-SOLA, F.J.; GAVILÁN, J.; LORENTE, N.; HUESO, J.J.; GONZÁLEZPADIERNA, C. M. Loquat fruit sink strength and growth pattern. Scientia Horticulturae, Wageningen, v. 98, p. 131-137, 2003.

DEMEY, J.; ZAMBRANO, A.; MACCHIAVELLI, R.; GONZÁLEZ, V. Tamaño de muestra en experimentos biotecnológicos con suspensiones celulares. Interciencia, Caracas, v.29, p. 396 - 400, 2004.

DE SILVA, H.N.; BALL, R.D. Mixed model analysis of within-plant variation of fruit weight with implications for sampling of kiwifruit vines. Annals of Botany, London, v.79, p.411-418, 1997.

DE SILVA, H.N.; HALL, A.J.; CASHMORE, W.M.; TUSTIN, D.S. Variation of fruit size and growth within an apple tree and its influence on sampling methods for estimating the parameters of mid-season size distributions. Annals of Botany, London, v. 86, p. 493- 501, 2000.

GOMEZ, K.A.; GOMEZ, A.A. Statistical procedures for agricultural research. $2^{\text {nd }} e d$. Ames: John Wiley \& Sons, 1984. 680 p.

MILES, D.B.; SMITH, G.S.; MILLER, S.A. Within plant sampling procedure- Fruit variation in Kiwifruit vines. Annals of Botany, London, v.78, p.289- 294, 1996.

MONTGOMERY, D.C. Design and analysis of experiments. $2^{\text {nd }}$ ed. London: John Wiley \& Sons, $1981.535 \mathrm{p}$.

OPARA, L.U. Fruit growth measurement and analysis. Horticultural Reviews, New York, v.24, p.373-431, 2000 . 
RAZONE, D.E.; NATALE, W.; PRADO, R.; BARBOSA, J.C. Amostragem para diagnose do estado nutricional de mangueiras. Revista Brasileira de Fruticultura, Jaboticabal, v.29, n.2, p.371-376, 2007.

SAS INSTITUTE. SAS/STAT ${ }^{\circledR}$ 9.1 user's guide. Cary: SAS INSTITUTE, 2004.

SAS INSTITUTE. SAS/STAT Versión 8. Site: 0014759001. Cary: SAS INSTITUTE, 2000.
SCHEAFFER, R.L.; MENDENHALL III, W.; LYMAN OTT, R. Elementos de muestreo. $6^{\text {th }}$ ed. Madri: International Thomson Editors, 2007. 455 p.

VILELA LOPES, M.; LEITE OLIVERA, C.A.; BARRETO, M.; BARBOSA, J.C.; ROSSI, F.M. Dimensionamento de amostras para monitoramento do ácaro da leprose Brevipalpus phoenicis (Geijskes, 1939) em citros. Revista Brasileira de Fruticultura, Jaboticabal, v.29, n.3, p.671-676, 2007. 